\title{
Separable balls around the maximally mixed multipartite quantum states
}

\author{
Leonid Gurvits and Howard Barnum \\ CCS-3, Mail Stop B256, Los Alamos National Laboratory, Los Alamos, NM 87545
}

(Dated: February 12, 2003)

\begin{abstract}
We show that for an $m$-partite quantum system, there is a ball of radius $2^{-(m / 2-1)}$ in Frobenius norm, centered at the identity matrix, of separable (unentangled) positive semidefinite matrices. This can be used to derive an $\epsilon$ below which mixtures of $\epsilon$ of any density matrix with $1-\epsilon$ of the maximally mixed state will be separable. The $\epsilon$ thus obtained is exponentially better (in the number of systems) than existing results. This gives a number of qubits below which NMR with standard pseudopure-state preparation techniques can access only unentangled states; with parameters realistic for current experiments, this is 23 qubits (compared to 13 qubits via earlier results). A ball of radius 1 is obtained for multipartite states separable over the reals.
\end{abstract}

PACS numbers: 03.65.Ud,03.67.-a,03.67.Lx

\section{INTRODUCTION}

Entanglement is an important quantum resource, useful in quantum computation, cryptography, and communication protocols. Entangled quantum states are those that cannot be expressed as a mixture of product states. That is, if $\rho$ is an entangled state of $m$ systems (an " $m$ partite state"), there is no way to choose probabilities $p_{i}$ and states $\rho_{i}^{1}, \ldots, \rho_{i}^{m}$ for systems 1 through $m$, such that

$$
\rho=\sum_{i} p_{i} \rho_{i}^{1} \otimes \cdots \otimes \rho_{i}^{m} .
$$

In this paper, we provide a simple geometric condition sufficient to guarantee separability (non-entanglement) of an $m$-partite state: that the state is proportional to the identity matrix plus a Hermitian perturbation $\Delta$ whose Frobenius norm (2-norm) is no greater than $2^{-(m / 2-1)}$. This is exponentially better than the best previous bounds we are aware of 1, 2]. It can be used to obtain balls of normalized separable states. Because the set of separable (unentangled) density matrices is convex, and the size of the largest ball that fits inside it (as well as the smallest ball that covers it) is important to complexity-theoretic questions involving convex sets, we expect the result to have applications in complexity questions about entanglement, such as the complexity of deciding whether or not a multipartite state is entangled. Equally importantly, it can help determine whether or not entanglement is present in interesting theoretical and experimental situations. For example, though the utility of the criterion is emphatically not restricted to such states, it gives us a bound on the "polarization" $\epsilon$ below which "pseudopure" states of the form $(1-\epsilon) I / d+\epsilon \pi$, with $\pi$ pure, $I$ the identity operator on a multipartite state-space of overall dimension $d$ are separable. Applied to a standard nuclear magnetic resonance (NMR) quantum information processing (QIP) protocol, pseudopurestate liquid NMR, at 300 Kelvin and a 11 Tesla external field, it tells us that unless we have 23 or more nuclearspin qubits, only separable states can be produced (compared to 13 qubits using the bound in [1]).
The methods of 1, 2] are very different from ours: they expand the density matrix in an overcomplete basis of pure states, and find conditions guaranteeing positivity of all coefficients of the expansion, thus giving an explicit decomposition of the form (1). In contrast, the methods we use have a nonconstructive flavor: although they establish that any $m$-partite (unnormalized) density matrix within a distance $1 / 2^{(m / 2-1)}$ is separable, they do not provide an explicit "separable representation" (1) of it. Our methods use general concepts of matrix theory and convex analysis in terms of which the problem is naturally formulated, and involve only short and elementary calculations. This paper is a natural sequel to [3], in the sense that almost the same mathematics is used. The main (though quite simple) novelty here is a generalization of separability, so-called $\left(C_{1} \otimes C_{2} \otimes \ldots \otimes C_{m}\right)$-separability, where $C_{i}$ are matrix cones (see Definition 2 below). This generalization arises naturally in extending the bipartite result of [3] to $m$-partite systems with $m \geq 3$. A reader comfortable with the technique used in [3] should have no extra problems in understanding this paper.

\section{NOTATION AND MATHEMATICAL PRELIMINARIES}

The mathematical notion of a "regular" positive cone (which we will just call cone) is basic in quantum information science, especially in the study of entanglement. This is so because the unnormalized quantum states, the unnormalized separable states of a multipartite quantum system, the completely positive maps, the positive maps, and many other sets of interest form such cones. (Appropriate normalization-like conditions, such as unit trace for states, or trace-preservation or trace-nonincrease for maps, are usually just additional linear equalities or inequalities.) In this section, we review regular positive cones and related notions; background and preliminaries specific to the separable cones (of unentangled states) appear at the beginning of the next section.

Definition $1 A$ positive cone is a subset $K$ of a real vector space $V$ closed under multiplication by positive 
scalars. It is called regular if it is (a) convex (equivalently, closed under addition: $K+K=K$ ), (b) generating $(K-K=V$, equivalently $K$ linearly generates $V$,) (c) pointed ( $K \cap-K=\emptyset$, so that it contains no nonnull subspace of $V$ ), and (d) topologically closed (in the Euclidean metric topology, for finite dimension).

Such a positive cone induces a partial order $\geq_{K}$ on $V$, defined by $x \geq_{K} y:=x-y \in K$. It is "linearcompatible": inequalities can be added, and multiplied by positive scalars. A set $S$ is said to generate a cone $K$ if $K$ is the set of positive linear combinations of elements of $S$. The topological closure condition guarantees that such a cone is generated (via addition) by its extreme rays. These are sets $R_{x}:=\{\lambda x: \lambda \geq 0\}$ such that no $y \in R_{x}$ can be written as a convex combination of elements of $C$ that are not in $R_{x}$. We will not make much use of closure and extremality, but at some points we use the fact that positive semidefinite (PSD) matrices can be written as convex combinations of rank-one PSD matrices (these being the members of the extreme rays of the cone of PSD matrices).

Duality is often a useful tool when dealing with cones. The dual vector space $V^{*}$ for real $V$ is the space of linear functions ("functionals") from $V$ to $\mathbf{R}$; the dual cone $C^{*} \subset V^{*}$ of the cone $C \subset V$ is the set of such linear functionals which are nonnegative on $C$. For finite dimensional vector spaces, $V^{*}$ is isomorphic to $V$ as a vector space (they have the same dimension). However, this isomorphism is not canonical; any nonsingular linear maps from $V$ onto $V^{*}$ does the job. For a slight improvement in clarity below, for $\alpha \in V^{*}, x \in V$, we write the value of $\alpha$ on $x$ as $\alpha[x]$, rather than $\alpha(x)$.

We define the adjoint $\phi^{\dagger}: V_{2}^{*} \rightarrow V_{1}^{*}$ of a linear map $\phi: V_{1} \rightarrow V_{2}$ via

$$
\phi^{\dagger}(\alpha)[x]=\alpha[\phi(x)],
$$

for all $\alpha \in V^{*}, x \in V$.

An inner product, written $\langle$,$\rangle , on V$ distinguishes a particular isomorphism $\zeta$ between $V$ and $V^{*}$, defined by requiring that $\zeta(x) \in V^{*}$ satisfy $\zeta(x)[y]=\langle x, y\rangle$, for all $y \in V$. From now on, we will assume such a choice of inner product (there will be a natural choice in our applications), and canonically identify $V$ with $V^{*}$ via $\zeta$. Thus we will view the dual cone $C^{*}$ as $\{y \in V:\langle y, x\rangle \geq$ $0, \forall x \in C\}$, and the adjoint of $\phi: V_{1} \rightarrow V_{2}$ as $\phi^{\dagger}: V_{2} \rightarrow$ $V_{1}$ defined via

$$
\langle B, \phi(A)\rangle=\left\langle\phi^{\dagger}(B), A\right\rangle,
$$

for all $A \in V_{1}, B \in V_{2}$.

We say a linear map $\phi: V_{1} \rightarrow V_{2}$ is $C_{1}$-to- $C_{2}$ positive, for cones $C_{1} \subset V_{1}, C_{2} \subset V_{2}$, if $\phi\left(C_{1}\right) \subseteq C_{2}$. Under either definition of $\phi^{\dagger}$, the following proposition is easily (but instructively) verified.

Proposition 1 If $\phi\left(C_{1}\right) \subseteq C_{2}$ then $\phi^{\dagger}\left(C_{2}^{*}\right) \subseteq C_{1}^{*}$.
The positive semidefinite cone $P S D(d)$ in the real linear space of Hermitian $d \times d$ matrices, is the set of matrices $M$ such that $x^{\dagger} M x \geq 0$ for all $x \in \mathbf{C}^{d}$. It is self-dual; if we use the trace inner product $\langle X, Y\rangle:=\operatorname{tr} X Y$ to identify $V^{*}$ with $V$, it is not only isomorphic to, but equal to, its dual. We will denote by " $\succeq$ " the partial order induced by this cone, and often write $M \succeq 0$ for the equivalent $M \in P S D(d)$.

We will have several occasions to use the following proposition, which follows from the fact that for normal (including Hermitian) matrices, $\Delta,\|\Delta\|_{\infty}$ is the largest modulus of an eigenvalue of $\Delta$ ).

Proposition 2 Let $\Delta$ be Hermitian. Then $I+\Delta \succeq 0$ is equivalent to $\|\Delta\|_{\infty} \leq 1$.

\section{SEPARABLE CONES}

Let us consider an $m$-partite unnormalized density matrix (i.e. just positive semidefinite)

$$
\rho: H_{1} \otimes H_{2} \otimes \ldots \otimes H_{m} \longrightarrow H_{1} \otimes H_{2} \otimes \ldots \otimes H_{m}
$$

Let $\operatorname{dim}\left(H_{i}\right)=d_{i}, 1 \leq i \leq m$. Then any such

$\left.\rho=\left\{\rho\left(i_{1}, i_{2}, \ldots, i_{m} ; j_{1}, j_{2}, \ldots, j_{m}\right)\right\}, 1 \leq i_{k}, j_{k} \leq d_{k} ; 1 \leq k \leq m\right\}$.

Let us block-partition $\rho$ with respect to the first index :

$$
\rho:=\left(\begin{array}{cccc}
\rho^{1,1} & \rho^{1,2} & \ldots & \rho^{1, d_{1}} \\
\rho^{2,1} & \rho^{1,2} & \ldots & \rho^{2, d_{1}} \\
\ldots & \ldots & \ldots & \ldots \\
\rho^{d_{1}, 1} & \rho^{d_{1}, 2} & \ldots & \rho^{d_{1}, d_{1}}
\end{array}\right) .
$$

where the blocks

$$
\rho^{i, j}: H_{2} \otimes \ldots \otimes H_{m} \longrightarrow H_{2} \otimes \ldots \otimes H_{m}
$$

and

$\rho^{i, j}=\left\{\rho\left(i, i_{2}, \ldots, i_{m} ; j, j_{2}, \ldots, j_{m}\right\}, 1 \leq i_{k}, j_{k} \leq d_{k} ; 2 \leq k \leq m\right\}$.

Definition 2 The linear space of $N \times N$ complex matrices is denoted $M(N)$, the linear space over the reals of $N \times N$ real matrices is denoted Mat $(N)$, the linear space of real symmetric $N \times N$ matrices is denoted $R \operatorname{Sym}(N)$, the linear space over reals of $N \times N$ complex hermitian matrices is denoted as $\mathrm{Her}(N)$. The space of complex block matrices, $K$ blocks by $K$ blocks, with blocks in $M(N)$, is denoted $\operatorname{Block}(K, N)$

Consider cones $C_{i} \subset M\left(d_{i}\right), 1 \leq i \leq m$. A matrix

$$
\rho: H_{1} \otimes H_{2} \otimes \ldots \otimes H_{m} \longrightarrow H_{1} \otimes H_{2} \otimes \ldots \otimes H_{m}
$$

(i.e. $\left.\rho \in M\left(d_{1} d_{2} \ldots d_{m}\right)\right)$ is called $\left(C_{1} \otimes C_{2} \otimes \ldots \otimes C_{m}\right)$ separable if it belongs to the cone generated by the set $\left\{A_{1} \otimes A_{2} \otimes \ldots \otimes A_{m}: A_{i} \in C_{i}, 1 \leq i \leq m\right\}$. 
This is trivially equivalent to the recursive definition: $\operatorname{Sep}\left(C_{1}, C_{2}, \ldots, C_{m}\right)$ is the cone generated by the pairs $A_{1} \otimes B$ with $A_{i} \in C_{1}, B \in \operatorname{Sep}\left(C_{2}, \ldots, C_{m}\right)$.

Examples: 1. Let for all $1 \leq i \leq m$ the cone $C_{i}$ be the cone of positive semidefinite matrices, denoted by $P S D\left(d_{i}\right)$. In this case the definition of $\left(C_{1} \otimes C_{2} \otimes\right.$ $\left.\ldots \otimes C_{m}\right)$-separability is equivalent to the standard notion of separability of multiparty unnormalized density matrices. We will denote the corresponding cone of separable multiparty unnormalized density matrices as $\operatorname{Sep}\left(d_{1}, d_{2}, \ldots, d_{m}\right)$.

2. Let for all $1 \leq i \leq m$ the cone $C_{i}$ be the cone of positive semidefinite matrices with real entries. We call the the corresponding cone the cone of realseparable multiparty density matrices and denote it by $R \operatorname{Sep}\left(d_{1}, d_{2}, \ldots, d_{m}\right)$.

We now recursively define a subspace $R \operatorname{Lin}\left(d_{1}, \ldots, d_{m}\right)$, which we show is the minimal linear subspace (over the reals) of the symmetric matrices $R \operatorname{Sym}\left(d_{1}, \ldots, d_{m}\right)$ that contains the real separable cone $R \operatorname{Sep}\left(d_{1}, \ldots, d_{m}\right)$.

Definition $3 \rho \in R \operatorname{Lin}\left(d_{1}, d_{2}, \ldots, d_{m}\right)$ iff in the block-

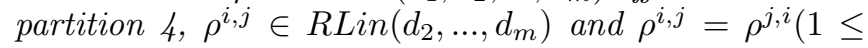
$\left.i, j \leq d_{1}\right) ; R \operatorname{Lin}(d)=R \operatorname{Sym}(d)$.

It is easy to prove that $\rho: H_{1} \otimes H_{2} \otimes \ldots \otimes H_{m} \longrightarrow$ $H_{1} \otimes H_{2} \otimes \ldots \otimes H_{m}$ is real-separable iff $\rho$ is separable and $\rho \in R \operatorname{Lin}\left(d_{1}, d_{2}, \ldots, d_{m}\right)$. In fact, we will show

Proposition $3 R \operatorname{Rin}\left(d_{1}, d_{2}, \ldots, d_{m}\right)$ is the minimal linear subspace (over the reals) of RSym $\left(d_{1} d_{2} \ldots d_{m}\right)$ which contains $R \operatorname{Sep}\left(d_{1}, d_{2}, \ldots, d_{m}\right)$.

Proof: It is clear that $R \operatorname{Lin}\left(d_{1} \ldots d_{m}\right)$ is a subspace of (" $\leq$ ") $R \operatorname{Sym}\left(d_{1} \ldots d_{m}\right)$. To be explicit, symmetry means $\rho\left(i_{1}, \ldots, i_{m}, j_{1}, \ldots, j_{m}\right)=\rho\left(j_{1}, \ldots, j_{m}, i_{1}, \ldots, i_{m}\right)$; this follows from the definition of RLin and $R \operatorname{Lin}\left(d_{2} \ldots d_{m}\right)$ 's being a subspace of $\operatorname{RSym}\left(d_{2}, \ldots, d_{m}\right)$. That establishes our induction step; the base case $R \operatorname{Lin}\left(d_{m}\right)=\operatorname{RSym}\left(d_{m}\right)$ clearly also holds.

Suppose $X \in R \operatorname{Sep}\left(d_{1}, \ldots, d_{m-1}\right)$. That is, $X=$ $\sum_{k} A_{k} \otimes B_{k}, A_{k} \in R \operatorname{Sep}\left(d_{1}, \ldots, d_{m-1}\right), B_{k} \in R \operatorname{Sym}\left(d_{m}\right)$. By the induction hypothesis, $A_{k} \in R \operatorname{Lin}\left(d_{1}, \ldots, d_{m-1}\right)$. Block-partitioning with respect to the second system,

$$
X^{i j}=\sum_{k} B_{k}(i, j) A_{k}=\sum_{k} B_{k}(j, i) A_{k}=X^{j i},
$$

These blocks are in $R \operatorname{Lin}\left(d_{1} \ldots d_{m-1}\right)$ because $A_{k}$ are; consequently $X \in R \operatorname{Lin}\left(d_{1}, \ldots, d_{m}\right)$. The base case is trivial: $R \operatorname{Sep}\left(d_{1}\right) \subseteq\left(R S e p\left(d_{1}\right) \cap R \operatorname{Rin}\left(d_{1}\right)\right)$ holds with equality because $R S e p\left(d_{1}\right) \equiv P S D\left(d_{1}\right)$ and $R \operatorname{Lin}\left(d_{1}\right)=$ $\operatorname{RSym}\left(d_{1}\right)$.

For the opposite direction, let $X \in$ $R \operatorname{Lin}\left(d_{1}, \ldots d_{m}\right), \operatorname{Sep}\left(d_{1}, \ldots, d_{m}\right)$. By separability,

$$
X=\sum_{k} A_{k} \otimes B_{k} \otimes \cdots \otimes Z_{k},
$$

where $A_{k} \in P S D\left(d_{1}\right), B_{k} \in P S D\left(d_{2}\right), Z_{k} \in P S D\left(d_{m}\right)$ (and no restriction to $m=26$ is intended!). Let
$A_{k}=A_{k}^{1}+i A_{k}^{2}$ with $A_{k}^{1}$ real symmetric, $A_{k}^{2}$ real skewsymmetric, and similarly for $B$. Substituting these in (6) and keeping only terms with an even number of imaginary factors (since $X \in R L i n$ ), and block-partitioning the matrix according to the first subsystem, each block has the form:

$$
\sum_{k} A_{k}^{1}(i, j) R_{k}+\sum_{k} A_{k}^{2}(i, j) S_{k}
$$

Thus $X=X_{1}+X_{2}$, where the first term is blocksymmetric, the second block-skew-symmetric. This second term must therefore be zero. By the recursive definition of RLin (and Sep) we have that, for each fixed value of $i, j, X^{i j}$ must be block-symmetric when partitioned according to the second ("B") system. This block is

$$
\begin{aligned}
& \sum_{k} A_{k}^{1}(i, j) B_{k}^{1}(m, n) C^{k} \otimes \cdots Z^{k} \\
+ & \sum_{k} A_{k}^{1}(i, j) B_{k}^{2}(m, n) C^{k} \otimes \cdots Z^{k}
\end{aligned}
$$

and again only the first component is nonzero. Proceeding thus through all the subsystems, all terms with a skew-symmetric factor must be zero and we have:

$$
X=A_{k}^{1} \otimes B_{k}^{1} \otimes \cdots Z_{k}^{1},
$$

with each of $A_{k}, B_{k}, \ldots Z_{k}$ real symmetric and positive semidefinite, i.e. $X \in R S e p$.

The next lemma gives a simple but very useful criterion for $P S D\left(d_{1}\right) \otimes C\left(d_{2}\right)$-separability for any cone $C\left(d_{2}\right)$ of Hermitian matrices. It is a mild generalization of the necessary and sufficient criterion (cf. [4], 5]) for ordinary $\left(C\left(d_{2}\right)=P S D\left(d_{2}\right)\right)$ bipartite separability, that every positive linear map, applied to one subsystem of the bipartite system (i.e. to every block of its block density matrix) gives a positive semidefinite matrix.

Definition 4 A linear operator $\phi: M\left(d_{2}\right) \longrightarrow M(N)$ is called $C\left(d_{2}\right)$-positive if $\phi\left(C\left(d_{2}\right)\right) \subset P S D(N)$. If $X$ is a block matrix as in (4), $X_{i, j} \in M\left(d_{2}\right)$ and $\phi: M\left(d_{2}\right) \longrightarrow$ $M(N)$ is a linear operator then we define

$$
\tilde{\phi}(X):=\left(\begin{array}{cccc}
\phi\left(X^{1,1}\right) & \phi\left(X^{1,2}\right) & \ldots & \phi\left(X^{1, d_{1}}\right) \\
\phi\left(X^{2,1}\right) & \phi\left(X^{2,2}\right) & \ldots & \phi\left(X^{2, d_{1}}\right) \\
\ldots & \ldots & \ldots & \ldots \\
\phi\left(X^{d_{1}, 1}\right) & \phi\left(X^{d_{1}, 2}\right) & \ldots & \phi\left(X^{d_{1}, d_{1}}\right)
\end{array}\right) .
$$

Lemma 1 Suppose that the cone $C\left(d_{2}\right) \subset \operatorname{Her}\left(d_{2}\right) \subset$ $M\left(d_{2}\right)$. Then $X$ is $P S D\left(d_{1}\right) \otimes C\left(d_{2}\right)$-separable iff $\tilde{\phi}(X) \succeq 0$ (i.e. is positive semidefinite) for all $C\left(d_{2}\right)$ positive linear operators $\phi: M\left(d_{2}\right) \longrightarrow M\left(d_{1}\right)$.

The proof uses the following proposition, which generalizes the duality between positive linear maps and separable states. 
Proposition 4 For Hermitian matrices $M \in$ $\operatorname{Block}\left(d_{1}, d_{2}\right)$, the following are equivalent:

1. $\operatorname{tr} M Z \geq 0$ for all $P S D\left(d_{1}\right) \otimes C\left(d_{2}\right)$-separable matrices $Z$.

2. $M^{i j}=\chi\left(e_{i} e_{j}^{\dagger}\right)$ for some $\chi$ such that $\chi\left(P S D\left(d_{1}\right)\right) \subseteq C\left(d_{2}\right)^{*}$.

Proof of Proposition 4 Item 1 of the proposition is equivalent to the same statement with $Z$ of the form $x x^{\dagger} \otimes Y$, with $x x^{\dagger}$ a rank-one matrix in $P S D\left(d_{1}\right)$ and $Y \in C\left(d_{2}\right)$. This is equivalent to:

$$
\sum_{i j} x_{i} x_{j}^{*} \operatorname{tr}\left(M^{i j} Y\right)=\operatorname{tr} \sum_{i j} x_{i} x_{j}^{*} M^{i j} Y \geq 0 .
$$

Since this holds for any $Y \in C\left(d_{2}\right)$, this says $\sum_{i} x_{i} x_{j}^{*} M^{i j} \in C\left(d_{2}\right)^{*}$. Define a linear map $T_{M}$ : $M\left(d_{1}\right) \rightarrow M\left(d_{2}\right)$ via $T_{M}\left(e_{i} e_{j}^{\dagger}\right)=M^{i j}$. Then for any $x \in \mathbf{C}^{d_{1}}, T\left(x x^{\dagger}\right)=\sum_{i j} x_{i} x_{j}^{*} T_{M}\left(e_{i} e_{j}^{\dagger}\right)=\sum_{i j} x_{i} x_{j}^{*} M^{i j}$, which we have just shown is in $C\left(d_{2}\right)^{*}$. Since $x x^{\dagger}$ generate $P S D\left(d_{1}\right)$, this is equivalent to saying $T_{M}$ takes $P S D\left(d_{1}\right)$ into $C\left(d_{2}\right)^{*}$, so $T_{m}$ is the desired $\chi$. The other direction $(2 \Rightarrow 1)$ is similar (most of the steps above were equivalences).

We remark that essentially the same argument establishes a similar statement for arbitrary pairs of cones of Hermitian matrices, in which $P S D\left(d_{1}\right)$ is replaced by a cone $C\left(d_{1}\right)$ in item 1 , and by $C\left(d_{1}\right)^{*}$ in item 2 .

Proof of Lemma 1: "Only if" is trivial: $P S D\left(d_{1}\right) \otimes$ $C\left(d_{2}\right)$-separability of $X$ means $X=\sum_{i} A_{i} \otimes B_{i}$, with $A_{i} \in P S D\left(d_{1}\right), B_{i} \in C\left(d_{2}\right)$, hence $\tilde{\phi}(X)=\sum_{i} A_{i} \otimes$ $\phi\left(B_{i}\right)$; since we assumed $\phi\left(C\left(d_{2}\right)\right) \subseteq P S D\left(d_{1}\right)$, we have $\phi\left(B_{i}\right) \in P S D\left(d_{1}\right)$, so $\tilde{\phi}(X) \in S e p\left(d_{1}, d_{1}\right)$.

For "if," note that $\tilde{\phi}(X) \succeq 0$ says that for any positive semidefinite $B \in \operatorname{Block}(N, \bar{K})$,

$$
\sum_{i j} \operatorname{tr} B^{i j} \phi\left(X^{i j}\right) \geq 0 .
$$

By the definition of dual cone (and the self-duality of $\left.P S D\left(d_{1}\right)\right)$ it is easily seen that $\phi^{\dagger}\left(P S D\left(d_{1}\right)\right) \subseteq C\left(d_{2}\right)^{*}$. Now, (12) is equivalent to

$$
\sum_{i j} \operatorname{tr} \phi^{\dagger}\left(B^{i j}\right) X^{i j}=\operatorname{tr} \tilde{\phi}^{\dagger}(B) X \geq 0 .
$$

Letting $B^{i j}=e_{i} e_{j}^{\dagger}$, so $B$ is the block matrix of a positive semidefinite rank-one state (specifically, the unnormalized maximally entangled state $x x^{\dagger}$ with $x=\sum_{i} e_{i} \otimes e_{i}$ ), we have that the matrix $\tilde{\phi}^{\dagger}(B)$ satisfies condition 2 of the Proposition; as $\phi$ ranges over all $P S D\left(d_{1}\right)$-to- $C\left(d_{2}\right)^{*}$ positive maps, $\tilde{\phi}^{\dagger}(B)$ ranges over all such matrices so by Proposition $4 X$ is $P S D\left(d_{1}\right) \otimes C\left(d_{2}\right)$-separable.

The next proposition will allow us to extend the (exact) bipartite result from [3] to multiparty systems. The bipartite result was that everything in the ball $B(N, 1):=$ $\left\{I+\Delta:\|\Delta\|_{2} \leq 1\right\}$ of size 1 in Frobenius norm around the identity operator is separable; therefore, so is everything in the cone, which we call $G\left(d_{1} d_{2}, 1\right)$, generated by that ball, for a bipartite system with subsytems of dimensions $d_{1}, d_{2}$. We define a slight generalization of this cone:

Definition 5 Let $G(N, a) \subset \operatorname{Her}(N) \subset M(N)$ be the cone generated by hermitian $N \times N$ matrices of the form $\left\{I+\Delta:\|\Delta\|_{2}=:\left(\operatorname{tr}\left(\Delta \Delta^{\dagger}\right)^{\frac{1}{2}} \leq a\right\}\right.$.

A sufficient condition for tripartite separability of $X$ is clearly that it belong to the cone generated by $A_{i} \otimes Z_{i}$, where $A_{i} \in P S D\left(d_{1}\right)$ and $Z_{i} \in G\left(d_{2} d_{3}, 1\right)$; this can be used to derive a tripartite sufficient condition for separability in terms of Frobenius norm. Letting this tripartite 2-norm ball generate a cone of separable tripartite states, similar reasoning gives a ball of 4-partite states, and so on. The key to the induction step is the following proposition.

Proposition 5 If $\phi: M(N) \longrightarrow M(K)$ is a $G(N, a)$ positive linear operator (i.e. $\phi(X) \succeq 0$ for all $X \in$ $G(N, a)$ and $\phi(I)=I \in M(K)$ then

$$
\begin{aligned}
& \text { 1. }\|\phi(X)\|_{\infty} \leq a^{-1}\|X\|_{2} \text { for all hermitian } X \in \\
& \text { M(N). } \\
& \text { 2. }\|\phi(Y)\|_{\infty} \leq a^{-1} \sqrt{2}\|Y\|_{2} \text { for all } Y \in M(N) .
\end{aligned}
$$

Proof: All $G(N, a)$-positive $\phi$ satisfy $\phi(I+\Delta) \succeq 0$ for all $\Delta$ such that $\|\Delta\|_{2} \leq a$, which when $\phi(I)=I$ gives $I+\phi(\Delta) \succeq 0$; by Proposition 2 this is equivalent to $\|\phi(\Delta)\|_{\infty} \leq 1$, establishing item 1. Item 2. uses item 1 and the following:

If a linear operator $\phi: M(N) \longrightarrow M(K)$ satisfies $\phi(\operatorname{Her}(N)) \subset \operatorname{Her}(K)$ and $\|\phi(Z)\|_{\infty} \leq\|X\|_{2}$ for all hermitian $Z \in M(N)$ then $\|\phi(Y)\|_{\infty} \leq \sqrt{2}\|Y\|_{2}$ for all $Y \in M(N)$.

To show this, let $Y=A+i B$ with $A, B$ Hermitian. Now, $\phi(Y)=\phi(A)+i \phi(B)$, so $\|\phi(Y)\|_{\infty} \leq\|\phi(A)\|_{\infty}+$ $\|\phi(B)\|_{\infty}$. This is less than or equal to $\|A\|_{2}+\|B\|_{2}$ by assumption ( $A, B$ being Hermitian). The conclusion follows by the square root of the elementary inequality $(x+y)^{2} \leq 2\left(x^{2}+y^{2}\right)$ (obtained from $2 x y \leq x^{2}+y^{2}$, which comes from $\left.(x-y)^{2} \geq 0\right)$.

The following example shows that the extra $\sqrt{2}$ factor is the best possible in this statement.

Consider $\phi: M(2) \longrightarrow M(2), \phi(X)=X(1,1) A_{1}+$ $X(2,2) A_{2}$; where $A_{1}, A_{2}$ are real symmetric anticommuting unitary matrices:

$$
\begin{aligned}
A_{1} & =\left(\begin{array}{cc}
1 & 0 \\
0 & -1
\end{array}\right), \\
A_{2} & =\left(\begin{array}{ll}
0 & 1 \\
1 & 0
\end{array}\right) .
\end{aligned}
$$

Notice that for real $a, b$ we have that $a A_{1}+b A_{2}=$ $\left(a^{2}+b^{2}\right)^{\frac{1}{2}} U$ for some real symmetric unitary $U$. Thus 
$\phi(H e r(2)) \subset \operatorname{Her}(2)$ and $\|\phi(Z)\|_{\infty} \leq\|Z\|_{2}$ for all hermitian $Z \in M(2)$. Consider the following (nonhermitian) matrix :

$$
Y=\left(\begin{array}{ll}
1 & 0 \\
0 & i
\end{array}\right)
$$

Then $\|Y\|_{2}^{2}=2$ and $\|\phi(Y)\|_{\infty}^{2}=\|\phi(Y)\|_{2}^{2}=4$, since $\operatorname{Det}(\phi(Y))=0$.

Problem: Is the extra $\sqrt{2}$ factor still the best possible if, additionally, $\phi(I)=I$ ?

Proposition 2 of [3] is a similar contraction result with constant 1 rather than $\sqrt{2}$ on all matrices, not just Hermitian ones, for the usual positive maps; the proofs used there do not work for the different notion of positivity used here.

Now everything is ready for our attack on multipartite separability.

Theorem 1 Let $H_{1}, H_{2}$ have dimensions $n_{1}, n_{2}$. If an unnormalized density matrix $\rho: H_{1} \otimes H_{2} \longrightarrow H_{1} \otimes H_{2}$ satisfies the inequality $\|\rho-I\|_{2} \leq a / \sqrt{2}$ then it is $P S D\left(n_{1}\right) \otimes G\left(n_{2}, a\right)$-separable.

\section{Proof:}

Let $\rho=I+\Delta \Delta$ Hermitian; by Lemma 1, we are looking for a bound on $\|\Delta\|_{2}$ that ensures, for any $G\left(n_{2}, a\right)$ to- $P S D\left(n_{1}\right)$-positive linear operator, that $\tilde{\phi}(I+\Delta) \succeq 0$. $\tilde{\phi}(I)=I$, so $\tilde{\phi}(I+\Delta)=I+\tilde{\phi}(\Delta) ;\|\tilde{\phi}(\Delta)\|_{\infty} \leq 1$ will ensure this (cf. Proposition 2). The argument establishing that $\|\tilde{\phi}(\Delta)\|_{\infty} \leq 1$ is essentially identical to the proof of the main theorem of [3] , except that because $\phi$ is not an ordinary positive map we must use the weaker contraction bound of Proposition 5 , with its $\sqrt{2}$ factor, in place of [3] 's result with a factor 1 .

$$
\|\tilde{\phi}(\Delta)\|_{\infty}^{2} \leq\|A\|_{\infty}^{2} \leq\|A\|_{2}^{2},
$$

where $A:=\left[a_{i j}\right], a_{i j}:=\left\|\phi\left(\Delta^{i j}\right)\right\|_{\infty}$.

$$
\|A\|_{2}^{2}=\sum_{i j} a_{i j}^{2}=\sum_{i j}\left\|\phi\left(\Delta^{i j}\right)\right\|_{\infty}^{2}
$$

(The first inequality is because the operator norm of a block matrix is bounded above by that of the matrix whose elements are the norms of the blocks, and the second is because the Frobenius norm is an upper bound to the operator norm.) $\left\|\phi\left(\Delta^{i j}\right)\right\|_{\infty}^{2} \leq 2 a^{-2}\left\|\Delta^{i j}\right\|_{\infty}^{2}$ by Proposition [5] and it is an elementary norm inequality that $\left\|\Delta^{i j}\right\|_{\infty} \leq\left\|\Delta^{i j}\right\|_{2}$. So

$$
\begin{aligned}
& \|\tilde{\phi}(\Delta)\|_{\infty}^{2} \leq 2 a^{-2} \sum_{i j}\left\|\phi\left(\Delta^{i j}\right)\right\|_{\infty}^{2} \\
& \leq 2 a^{-2} \sum_{i j}\left\|\Delta^{i j}\right\|_{2}^{2} \equiv 2 a^{-2}\|\Delta\|_{2}^{2} .
\end{aligned}
$$

Thus if $\|\Delta\|_{2} \leq a / \sqrt{2},\|\tilde{\phi}(\Delta)\|_{\infty} \leq 1$.
Corollary 1 If an m-partite unnormalized density matrix $\rho: H_{1} \otimes \cdots \otimes H_{m} \longrightarrow H_{1} \otimes \cdots \otimes H_{m}$ satisifes $\|\rho-I\|_{2} \leq 1 /\left(2^{m / 2-1}\right)$ then it is separable.

Proof: The main result of [3] is that $G\left(d_{1} d_{2}, 1\right) \subset$ $\operatorname{Sep}\left(d_{1}, d_{2}\right)$. This is the base case for an induction on the number of subsystems. For the induction step, fix $m>2$ and suppose as our induction hypothesis the Corollary holds for $m-1$, i.e. $G\left(d_{1}, \ldots, d_{m-1}, 2^{-((m-1) / 2-1)}\right) \subseteq \operatorname{Sep}\left(d_{1}, \ldots d_{l}\right)$. Theorem 1 tells us $G\left(d_{1}, \ldots d_{m}, 2^{-((m-1) / 2-1)} / \sqrt{2} \equiv 2^{-(m / 2-1)}\right)$ is $P S D\left(d_{m}\right) \otimes G\left(d_{1} \ldots d_{m-1}, 2^{-(m-1) / 2-1)}\right)$-separable, and therefore (by the induction hypothesis and the recursive definition of separability) separable.

If we had an analogue to Theorem 11 with $G\left(n_{1}, a\right)$ instead of $P S D\left(n_{1}\right)$, and some constant $\alpha$ replacing $1 / \sqrt{2}$, then we could get one over a polynomial instead of an exponential in Corollary 1] by recursively dividing systems into subsystems of more or less equal size, since this involves a logarithmic number of partitionings compared to splitting off one system at a time. The first step toward such a theorem would be to apply the characterization of $C\left(d_{1}\right) \otimes C\left(d_{2}\right)$-separability analogous to Proposition 4 (and discussed after that proposition above) to $G\left(d_{1}, a\right), G\left(d_{2}, a\right)$; the fact that neither of these cones is self-dual has so far proved an obstacle to our getting useful results along these lines.

Theorem 2 Consider $\rho \in R \operatorname{Lin}\left(d_{1}, \ldots, d_{m}\right)$. If $\| \rho-$ $I \|_{2} \leq 1$ then

$$
\rho=\sum a_{i} \rho^{(i)} \otimes \rho_{i}, a_{i} \geq 0
$$

where for all $i \rho_{1}^{(i)}$ is a real positive semidefinite $d_{1} \times d_{1}$ matrix; $\rho_{i} \in R \operatorname{Lin}\left(d_{2}, \ldots, d_{m}\right)$ and $\left\|I-\rho_{i}\right\|_{2} \leq 1$.

Proof: The proof goes essentially like that of Theorem 1 except that the blocks $\Delta^{i j}$ are Hermitian by Proposition 3. Consequently we may use item 2. rather than item 1. of Proposition [5] and obtain the larger radius ball.

Corollary 2 If $\rho \in R \operatorname{Lin}\left(d_{1}, \ldots, d_{m}\right)$ and $\|\rho-I\|_{2} \leq$ 1 then $\rho$ is real-separable. In other words the maximal separable ball in $R \operatorname{Lin}\left(d_{1}, \ldots, d_{m}\right)$ around the identity $I$ has radius 1 .

The next Proposition is immediate from results of [3], derived using "scaling," i.e., considering all ways of writing a matrix $\rho$ as a positive scalar times the sum of the identity and a Hermitian perturbation, and minimizing the 2-norm of the perturbation).

Proposition 6 Define $\mu(\rho)$ as the maximum of $\|\Delta\|_{2}$ over all $\Delta$ such that there exists an $\alpha>0$ for which $\rho=$ $\alpha(I+\Delta)$. Let $\rho$ be a normalized $(\operatorname{tr} \rho=1)$ density matrix. Then the following three statements are equivalent:

1. $\mu(\rho) \leq a$.

2. $\operatorname{tr} \rho^{2} \leq 1 /\left(d-a^{2}\right)$.

3. $\|\rho-I / d\|_{2} \leq a / \sqrt{d\left(d-a^{2}\right)}$. 
Using this Proposition, Theorem 1 has (via Corollary (1) the following corollary:

Corollary 3 If an m-partite normalized (i.e. unit trace) density matrix $\rho: H_{1} \otimes \cdots \otimes H_{m} \longrightarrow H_{1} \otimes \cdots \otimes H_{m}$ satisifes $\|\rho-I / d\|_{2} \leq \frac{1}{2^{m / 2-1 d}}$, where $d=\operatorname{dim}\left(H_{1} \otimes\right.$ $\left.\cdots \otimes H_{m}\right)$, then it is separable.

(The proposition actually gives the (negligibly) tighter statement with $2^{m / 2-1} \sqrt{d\left(d-2^{-(m-2)}\right)}$ in the denominator.)

\section{DISCUSSION}

In many interesting experimental or theoretical situations, the system is in a "pseudopure state": a mixture of the uniform density matrix with some pure state $\pi$ :

$$
\rho_{\epsilon, \pi}:=\epsilon \pi+(1-\epsilon) I / d,
$$

where $d=d_{1}, . . d_{m}$ is the total dimension of the system. For example, consider nuclear magnetic resonance (NMR) quantum information-processing (QIP), where $d=2$ (the Hilbert space of a nuclear spin), and $m$ is the number of spins addressed in the molecule being used. As discussed in more detail below, the initialization procedures standard in most NMRQIP implementations prepare pseudopure states.

Write

$$
\rho_{\epsilon, \pi}=(1 / d) I+\epsilon(\pi-I / d) .
$$

Since $\|\epsilon(\pi-I / d)\|_{2}=\epsilon \sqrt{\frac{d-1}{d}}$, by Corollary 3 , this is separable if

$$
\epsilon \leq 2^{-(m / 2-1)} / \sqrt{d(d-1)},
$$

For $m D$-dimensional systems (so $d=D^{m}$ ), this implies the (negligibly loosened) bound

$$
\epsilon \leq 2^{-(m / 2-1)} / D^{m} .
$$

This is an exponential improvement over the result in [2] (the qubit case is in [1]) of $\epsilon \leq 1 /\left(1+D^{2 m-1}\right)$. For $m$ qubits, for example, our result goes asymptotically as $2^{-((3 / 2) m-1)}$, versus $2^{-(2 m-1)}$ in [1]. Another comparison is with our earlier bound of

$$
\epsilon \leq 1 /\left(D^{m}-1\right)
$$

guaranteeing separability for $m D$-dimensional systems with respect to every bipartition [3]. It is interesting that this is exponentially larger than the present bound guaranteeing multipartite separability, although we do not know that a tight multipartite bound would still exhibit this exponential separation.

In liquid-state NMR at high temperature $T$, the sample is placed in a high DC magnetic field. Each spin is in a highly mixed thermal state. It is not maximally mixed because of the energy splitting between the higherenergy state in which the spin is aligned with the magnetic field, and the higher-energy one in which it is antialigned. This gives probabilities for those states proportional to the Boltzmann factors $e^{ \pm \beta \mu B}$, where $\beta \equiv 1 / k T$ with $k$ Boltzmann's constant, $\mu$ the magnetic moment of the nuclear spin, $B$ the external field strength. For realistic high-T liquid NMR values of $T=300$ Kelvin, $B=11$ Tesla, $\beta \mu B \approx 3.746 \times 10^{-5} \ll 1$. Calling this $\eta$, $e^{ \pm \eta} \approx 1 \pm \eta$, so the probabilities are $p_{\uparrow} \approx(1-\eta) / 2, p_{\downarrow} \approx$ $(1+\eta) / 2$, where $\uparrow / \downarrow$ denote alignment/anti-alignment of the spin with the field. With independent, distinguishable nuclear spins, Maxwell-Boltzmann statistics give the highest-probability pure state, with all $m$ spins up (fieldaligned), probability about $(1+\eta)^{m} / 2^{m} \approx(1+m \eta) / 2^{m}$. Standard pseudopure-state preparation creates a mixture of this most probable pure state and the maximally mixed state, by applying a randomly chosen unitary from the group of unitaries fixing the all-spins-aligned state. ( $U$ could be chosen uniformly (i.e. with Haar measure on this group), but efficient randomization procedures may draw from carefully chosen finite sets of such unitaries [6].) Thus, we get a mixture

$$
(1-\epsilon) I / 2^{m}+\epsilon|\uparrow \cdots \uparrow\rangle \uparrow \cdots \uparrow \mid,
$$

with

$$
\epsilon=\eta m / 2^{m} .
$$

With $\eta \approx 3.746 \times 10^{-5}$. this implies that below about 23 qubits, NMR pseudopure states are all separable, compared to the $\approx 13$ qubits one gets from the bound in [1].

Even without entanglement considerations, it is clear that pseudopure-state NMR quantum computing will not give asymptotic gains over classical computing, because of the exponentially increasing signal-to-noise ratio from (24). As pointed out in 7], it does not follow that no application of liquid-state NMR can have better-than-classical asymptotic performance: NMRQIP is not limited to pseudopure-state initialization. It is not known that NMRQIP with other initialization schemes, such as those that involve preparing a fixed number of pseudopure qubits as the total number of spin qubits grows, can be efficiently classically simulated, and even with one pure qubit interesting things can be efficiently done for which no efficient classical algorithm is currently known [7]. Another non-pseudopure initialization scheme is the Schulman-Vazirani algorithmic cooling procedure 8], which essentially uses an efficient (and NMR-implementable) compression algorithm to convert the thermal state with entropy $S$, for $m$ nuclear spins, into $\log S$ maximally mixed qubits and $m-\log S$ pure ones. Their work shows that the theoretical model derived from NMR with an initial thermal state is as powerful as standard quantum computation. Though the overhead required is polynomial, the space overhead is 
large enough to be impractical given the small number of qubits available in liquid-state NMR. But algorithmic cooling is certainly relevant in principle to the asymptotic power of an implementation, and could be practically relevant to a high temperature bulk QIP implementation that was expected to be sufficiently scalable that asymptotic considerations are relevant.

However, there is still the interesting possibility that one may produce an entangled overall density matrix (and not just a mixture of the maximally mixed state with an entangled state) via pseudopure-state NMRQIP. The results herein increase the number of qubits known to be required before this may be possible, although, since we have not shown that the bounds herein are tight, with our assumed $\eta$ even at 23 qubits there is no guarantee one can prepare an entangled pseudopure state. By contrast, from (22) and (24) one needs $m=1 / \eta$ qubits (about 26,700 for our $\eta$ ) to have any hope of obtaining a pseudopure state that is not bipartite-separable with respect to a partition of the qubits into two sets. Again, we remind the reader that non-pseudopure protocols could conceivably give such a "biseparable" state with far fewer qubits; in the Appendix we discuss some implications of our results for this possibility.

In conclusion we have derived an upper bound, exponentially better than those already known, on the Frobenius-norm radius of a ball of separable matrices around the identity matrix. The bound has implications for the minimum polarization needed for bulk quantuminformation-processing protocols initialized by preparing pseudopure states from a thermal state by via averaging, to produce entanglement: known lower bounds on this minimal polarization are exponentially increased by our results. We stress, however, that this is just one application of a general, computationally simple sufficient criterion for multipartite entanglement, applicable to states of any form. Its geometric nature should make it useful in many applications, both theoretical and practical. The question of whether this bound is tight, or whether there is a larger separable Frobenius norm ball around the identity, remains open.

\section{APPENDIX A: ENTANGLEMENT AND THERMAL INITIAL STATES IN NMR}

Schulman and Vazirani's algorithmic cooling protocol shows that it is, in theory, possible to prepare any entangled state from sufficiently many thermal NMR qubits. The question of just how many qubits are required by means possibly simpler than algorithmic cooling is also of interest. One can gain some information about this using our results, by applying Corollary 3 to the initial thermal density matrix of an NMR system. This matrix, which is approximately

$$
\left(\begin{array}{cc}
\frac{1+\eta}{2} & 0 \\
0 & \frac{1-\eta}{2}
\end{array}\right)^{\otimes n}
$$

(with each qubit expressed in the $|\uparrow\rangle,|\downarrow\rangle$ basis), has

$$
\|\rho-I / d\|_{2}^{2}=\frac{\left(1+\eta^{2}\right)^{m}-1}{2^{m}} \approx m \eta^{2} / 2^{m} .
$$

This should be compared to Corollary 3's separability condition, which guarantees separability if this squared distance is below $2^{-(3 m-2)}$. The comparison gives that for $m$ qubits, the thermal state (and any state reachable from it by unitary transformation) is separable if

$$
\eta \leq m^{-1 / 2} 2^{m-1} .
$$

For the same experimental conditions considered above, 14 qubits are required before this bound is exceeded (rather than the 23 for the pseudopure state prepared from this thermal state).

If one wants the possibility of bipartite entanglement with respect to some partition of the qubits into two sets, it is necessary to beat the bound (closely related to (22))

$$
\|\rho-I / d\|_{2}^{2} \leq \frac{1}{d(d-1)} .
$$

For the thermal state, this gives $\eta \leq m^{-1 / 2} 2^{-m / 2}$. With $\eta=3.746 \times 10^{-5}$ as before, the bound is not surpassed until 25 qubits. Although this comes from an essentially tight bound, that does not imply that entanglement can be achieved through computation starting with this initial state. Although the thermal state has the same magnitude perturbation as some entangled state, the latter will have different eigenvalues, so unitary manipulation will not get us there, and it is an interesting question what we can achieve along these lines using NMR-implementable nonunitary manipulations (which may sometimes require extra thermal ancilla bits that should be counted as resources). To understand when unitary manipulations might be guaranteed to give us entanglement, a promising approach is to look for conditions on the spectrum of a matrix sufficient for an entangled state with that spectrum to exist. We conjecture that the problem of determining, from a spectrum, whether or not an entangled state with that spectrum exists, is NP-hard in terms of an appropriate measure of problem size; this does not rule out easier-to-evaluate sufficient conditions, perhaps obtained from relaxations of the above problem. Theorem 4 of [3] gives some information on spectra sufficient to guarantee entanglement.

For the pseudopure fraction $\epsilon$ there is an upper bound of $2 /\left(2+2^{m}\right)$ from [3] nearly matching the lower bound (22). Comparison to the expression $\epsilon=\eta m / 2^{m}$ for pseudopure polarization suggests that, at some large number of qubits $m \geq 2 / \eta$, even pseudopure protocols will exceed this bound. With $\eta=3.746 \times 10^{-5}$ this gives about 53,400 qubits. This is far beyond the range generally viewed as relevant for liquid state NMR, and improved polarization is unlikely to bring it into this range. Since the state demonstrating entanglement at the upper bound is not in general pseudopure, exceeding this 
bound is still no guarantee we can get entanglement. Also, this is well into the range where algorithmic cooling could produce much stronger entanglement. Still, one can imagine that in other bulk QIP implementations the balance between the difficulty of implementing complex unitaries (relatively easy in NMR) and the difficulty of preparing large numbers of thermal qubits (apparently relatively hard in NMR) could be different, and entanglement generation by simple manipulations on thermal states, perhaps even by pseudopure state preparation, might be promising.

\section{Acknowledgments}

We thank Manny Knill and Isaac Chuang for discussions, and the US DOE and NSA for financial support.
[1] S. Braunstein, C. M. Caves, R. Jozsa, N. Linden, S. Popescu, and R. Schack, Physical Review Letters 83, 1054 (1999).

[2] P. Rungta, W. J. Munro, K. Nemoto, P. Deuar, G. J. Milburn, and C. M. Caves, in Directions in Quantum Optics: A Collection of Papers Dedicated to the Memory of Dan Walls, edited by D. Walls, R. Glauber, M. Scully, and H. Carmichael (Springer, New York and Berlin, 2001), also arXiv.org e-print quant-ph/0001075

[3] L. Gurvits and H. Barnum, Physical Review A 66, 062311 (2002).

[4] S. L. Woronowicz, Reports on Mathematical Physics 10, 165 (1976).
[5] M. Horodecki, P. Horodecki, and R. Horodecki, Phys. Lett. A 223, 1 (1996), (also arXiv.org e-print quant-ph/9605038).

[6] E. Knill, I. Chuang, and R. Laflamme, Physical Review A 57, 3348 (1998).

[7] E. Knill and R. Laflamme, Physical Review Letters 81, 5672 (1998).

[8] L. J. Schulman and U. Vazirani, Proceedings of the 31st Annual ACM Symposium on the Theory of Computing (STOC) pp. 322-329 (1999), earlier version is quant-ph/9804060 